\title{
Artículos
}

\section{Las universidades públicas españolas en Google Scholar: presencia y evolución de su publicación académica web}

\author{
Por Enrique Orduña-Malea, Jorge Serrano-Cobos y Nuria Lloret-Romero
}

\begin{abstract}
Resumen: Se estudia la validez de Google Scholar como servicio que refleja adecuadamente la producción científica de una universidad, comparando su cobertura de las universidades públicas españolas con la mostrada por Scopus. Se analiza la presencia y evolución de la documentación científica de las sedes web de estas universidades desde enero hasta julio (ambos meses incluidos) de 2009. Los resultados muestran que, pese a encontrar cierta interrelación en la productividad de las instituciones en Scopus y Scholar, entre ambos sistemas existen grandes diferencias en los resultados totales que invalidan a este último como un reflejo válido de la producción universitaria. Finalmente, la mayoría de las webs universitarias analizadas presentan un crecimiento positivo aunque moderado en Scholar, durante el período estudiado.
\end{abstract}

Palabras clave: Sedes web universitarias, Google Scholar, Producción científica, Universidades públicas españolas, Rankings de universidades.

Title: Spanish public universities in Google Schola

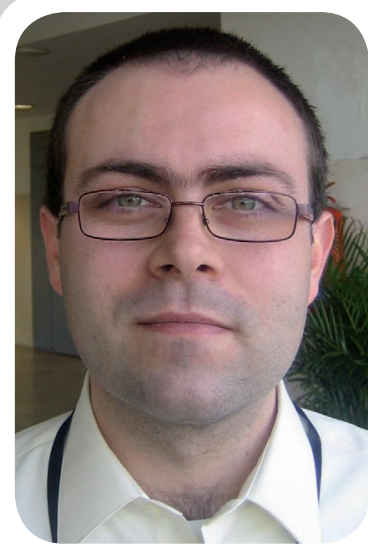

Enrique Orduña-Malea es ingeniero técnico de telecomunicaciones, esp. sonido e imagen, y técnico especialista en imagen fílmica, licenciado en documentación y máster en contenidos y aspectos legales en la sociedad de la información (especialidad en contenidos multicanal), por la Universidad Politécnica de Valencia (UPV). Investigador predoctoral en la división de investigación en tecnología e información (Calsi) del Instituto de Diseño y Fabricación (IDF) de la UPV. Redactor jefe del Anuario ThinkEPl, webmaster y miembro del thinktank sobre estudios de estrategia y prospectiva de la información, y miembro del equipo de trabajo del gestor social de noticias DocuMenea.

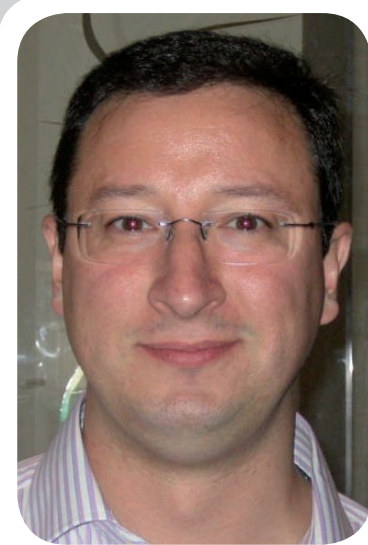

Jorge Serrano-Cobos, licenciado en documentación por la Univ. de Granada, ha trabajado como arquitecto de información y consultor en diseño de sistemas de información e intranets y especialista en marketing online para compañías como Planeta DeAgostini, Serikat Consultoría Informática y Google Inc. Es director del Departamento de Contenidos de MASmedios.com. Sus intereses profesionales van desde la arquitectura de información, el diseño de interacción (incluyendo opacs, juegos multimedia, etc.) al estudio de information seeking behaviour, web mining, search analytics, e-commerce y e-marketing. Miembro del think-tank sobre estrategia y prospectiva de la información ThinkEPI.

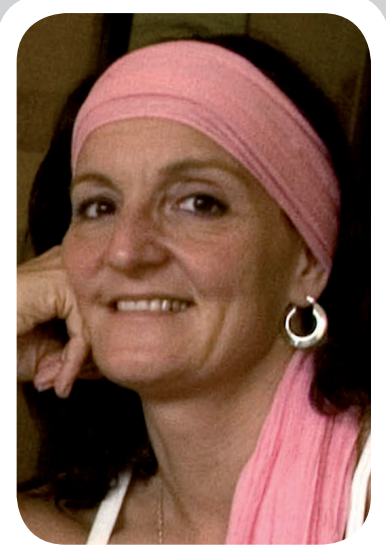

Nuria Lloret-Romero es doctora por la UPV, master en desarrollo de sist. de información online, soportes magnéticos cd-rom (Masdoc) de la UB, y master en dirección financiera de empresas (Fund. Estudios Bursátiles de Valencia). Prof titular y directora del Dpto. de Comunicación Audiovisual, Documentación e Historia del Arte (Dcadha) de la UPV. Subdirectora del Inst. de Investigación Univ. IDF y directora del grupo de investigación Calsi, de dicho Instituto. Evaluadora de la UE (programa IST). Socia fundadora de MASmedios, vicepres. de la Fed. Valenciana de empresas de Servicios Avanzados y asesora del Comité de Tecnologías de la Información de la Cámara de Comercio de Valencia.

Abstract: The validity of Google Scholar as a service that reflects properly the scientific output of a university is analyzed, comparing its coverage of Spanish public universities with that of Scopus. The presence and evolution of scientific documentation on the websites of the Spanish public universities from January to July (both months inclusive) 2009 is also studied. The results show that, despite finding some interrelationship between Scholar and Scopus concerning the productivity of institutions, there are large differences in the total results that override the latter as a valid reflection of university productivity. Finally, Scholar shows positive, albeit modest, growth during the studied period for most university websites analyzed.

Keywords: Academic websites, Google Scholar, Scientific output, Spanish public universities, University rankings.

Orduña-Malea, Enrique; Serrano-Cobos, Jorge; Lloret-Romero, Nuria. "La universidades públicas españolas en Google Scholar: presencia y evolución de su publicación académica web”. El profesional de la información, 2009, septiembre-octubre, v. 18, n. 5, pp. 493-500.

DOI: 10.3145/epi.2009.sep.02 


\section{Introducción}

La cibermetría como disciplina da un salto cuantitativo en 2004 con la puesta en marcha del Ranking web of world universities ${ }^{1}$, primer ranking de universidades de cobertura mundial basado en criterios meramente cibermétricos (Aguillo; Granadino; Ortega; Prieto, 2006; Aguillo; Ortega; Fernández, 2008).

Pese a la existencia de otros rankings ${ }^{2,3}$ y estudios acerca de la presencia web de las universidades mediante la información proporcionada por los buscadores (Bar-Ilan, 2004; Payne; Thelwall, 2004), esta iniciativa marca un hito debido a sus objetivos, cobertura, impacto mediático y orientación documental.

Tal como los responsables del proyecto expresan ${ }^{1}$, "la posición en el ranking resume el rendimiento global de la universidad, aporta información para estudiantes o profesores y refleja el compromiso académico con la difusión del conocimiento científico".

En este contexto, el concepto de "sede web académica" debe entenderse como un conjunto de webs ligadas jerárquicamente a una página principal, identificable por una url, y que forma una "unidad documental" reconocible e independiente de otras por su representatividad institucional (Aguillo, 1998).

Por tanto, asumiendo la web como un canal de comunicación entre la universidad y sus usuarios, la calidad y cantidad de documentación accesible desde su dominio web (que podrá alojar a una o más sedes web) serán indicadores de su grado de comunicación; siendo éste, a su vez, indicador parcial de su calidad como institución.

No obstante, la documentación publicada dentro de un dominio web académico es heterogénea, pudiendo ser clasificada fundamentalmente en administrativa, bibliográfica, científica, docente e informativa.

\section{"Google Scholar destaca por la inclusión de citas y por la mayor estabilidad de los resultados frente a los motores de búsqueda generalistas"}

La cantidad y calidad de cada uno de estos tipos de documentación sirven potencialmente para valorar, entre otros criterios, las diferentes misiones de la universidad (docencia, investigación y transferencia), o al menos la sub-misión de su puesta online.

Sin embargo, los motores de búsqueda no discriminan apropiadamente estas clases de documentación automáticamente ni proporcionan comandos adecua- dos para filtrarla de forma conveniente a nuestros propósitos (es decir, los ítems se encuentran etiquetados, pero no es posible filtrar por ellos), siendo este hecho delicado desde el punto de vista cienciométrico.

En 2006 se lanza Google Scholar con la intención de resolver algunos de estos problemas, así como de indizar la llamada internet invisible. Sus resultados pasan supuestamente un filtro por el que el sistema considera que son científicos, o se tienen al menos ciertas garantías. Esto se logra mediante acuerdos comerciales con proveedores, lo que explica en parte su cobertura.

De ser cierto significa que Scholar, con los comandos de búsqueda apropiados, podría ser usado como instrumento para medir la cantidad de documentación científica que las universidades publican en la Web y/o dentro su dominio web.

Esta última posibilidad permitiría medir parte del compromiso de comunicación entre la universidad y sus usuarios comentado anteriormente, dejando la documentación docente y de transferencia aparte. Este aspecto llevó a los responsables del ranking web a adoptar Google Scholar como uno de sus indicadores, a partir de su edición de 2006.

Google Scholar destaca sobre todo por la inclusión de citas (es la única base de datos multidisciplinar que las suministra, junto a Scopus y WoS) y por la mayor estabilidad de los resultados frente a los motores de búsqueda generalistas.

Aun así, se detecta una serie de inconvenientes en el uso de Scholar como herramienta bibliométrica (Torres-Salinas; Ruiz-Pérez; Delgado-López-Cózar, 2010):

- No todos los documentos indizados se pueden considerar académicos.

- No proporciona ninguna información sobre las fuentes que cubre.

- Cobertura disciplinar desigual en ciencias sociales y humanidades.

- Carencia de comandos básicos de consulta para realizar estudios bibliométricos.

- Ausencia absoluta de normalización en los datos (autores y fuentes).

A esto se unen otros problemas en la realización de análisis de citas (Jacsó, 2008a; Harzing; Van der Wal, 2008).

Este trabajo, por su parte, plantea otra serie de cuestiones relacionadas con la cobertura, visibilidad y evolución de los contenidos alojados en los dominios web de las universidades españolas: 


\begin{tabular}{|c|c|c|c|c|c|}
\hline Universidades & Código & url & Universidades & Código & url \\
\hline \multirow{2}{*}{$\begin{array}{l}\text { Universitat Autònoma } \\
\text { de Barcelona }\end{array}$} & \multirow{2}{*}{$U A B$} & http://www.uab.es/ & Universidad de Oviedo & UO & http://www.uniovi.es/ \\
\hline & & \multirow{2}{*}{ http://www.uam.es/ } & $\begin{array}{l}\text { Universidad de } \\
\text { Salamanca }\end{array}$ & USAL & http://www.usal.es \\
\hline $\begin{array}{l}\text { Universidad Autónoma } \\
\text { de Madrid }\end{array}$ & UAM & & \multirow{2}{*}{$\begin{array}{l}\text { Universidad de } \\
\text { Santiago de } \\
\text { Compostela }\end{array}$} & \multirow[b]{2}{*}{ USC } & \multirow[b]{2}{*}{ http://www.usc.es/ } \\
\hline $\begin{array}{l}\text { Universidad Carlos III } \\
\text { de Madrid }\end{array}$ & UC3M & http://www.uc3m.es & & & \\
\hline \multirow{2}{*}{$\begin{array}{l}\text { Universidad } \\
\text { Complutense de Madrid }\end{array}$} & \multirow{2}{*}{ UCM } & \multirow{2}{*}{ http://www.ucm.es/ } & Universidad de Sevilla & US & http://www.us.es \\
\hline & & & Universitat de València & UV & http://www.uv.es \\
\hline Universidad de Alcalá & $U A H$ & s/ & \multirow{2}{*}{$\begin{array}{l}\text { Universidad de } \\
\text { Valladolid }\end{array}$} & \multirow[b]{2}{*}{ UVA } & \multirow{2}{*}{ http://www.uva.es/ } \\
\hline Universitat d'Alacant & $U A$ & http://www.ua.es/ & & & \\
\hline Universidad de Almería & $U A L$ & http://www.ual.es/ & Universidad de Vigo & UVI & http://www.uvigo.es/ \\
\hline $\begin{array}{l}\text { Universitat de } \\
\text { Barcelona }\end{array}$ & $U B$ & http://www.ub.edu & \begin{tabular}{|l|} 
Universidad de \\
Zaragoza
\end{tabular} & $U Z$ & http://www.unizar.es/ \\
\hline Universidad de Burgos & $U B U$ & http://www.ubu.es & \multirow{2}{*}{$\begin{array}{l}\text { Universidad del País } \\
\text { Vasco }\end{array}$} & \multirow{2}{*}{$E H U$} & \multirow{2}{*}{ http://www.ehu.es } \\
\hline Universidad de Cádiz & $U C A$ & http://www.uca.es/ & & & \\
\hline $\begin{array}{l}\text { Universidad de } \\
\text { Cantabria }\end{array}$ & $U C$ & http://www.unican.es & $\begin{array}{l}\text { Universidad } \\
\text { Internacional de }\end{array}$ & UIA & http://www.unia.es/ \\
\hline \multirow{2}{*}{$\begin{array}{l}\text { Universidad de Castilla- } \\
\text { La Mancha }\end{array}$} & \multirow{2}{*}{$U C L M$} & \multirow{2}{*}{ http://www.uclm.es/ } & & & \\
\hline & & & & UJI & \\
\hline \multirow{2}{*}{$\begin{array}{l}\text { Universidad de } \\
\text { Extremadura }\end{array}$} & \multirow[b]{2}{*}{ UNEX } & \multirow[b]{2}{*}{ http://www.unex.es/ } & \multirow{3}{*}{\begin{tabular}{|l} 
Hernandez de Elche \\
Univ. Nacional de \\
Educación a Distancia \\
\end{tabular}} & $U M H$ & http://www.umh.es/ \\
\hline & & & & \multirow{2}{*}{ UNED } & \multirow{2}{*}{ http://www.uned.es } \\
\hline Universitat de Girona & $U D G$ & http://www.udg.edu/ & & & \\
\hline Universidad de Granada & $U G R$ & http://www.ugr.es & $\begin{array}{l}\text { Olavide } \\
\text { Olabio ae }\end{array}$ & UPO & http://www.upo.es/ \\
\hline Universidad de Huelva & $U H U$ & http://www.uhu.es/ & Universidad Politécnica & & \\
\hline Universidad de Jaén & UJA & http://www.ujaen.es/ & & UPCT & nttp://www.upct.es/ \\
\hline $\begin{array}{l}\text { Universidad de La } \\
\text { Coruña }\end{array}$ & $U D C$ & http://www.udc.es & $\begin{array}{l}\text { Universitat Politècnica } \\
\text { de Catalunya }\end{array}$ & $U P C$ & http://www.upc.es/ \\
\hline $\begin{array}{l}\text { Universidad de La } \\
\text { Laguna }\end{array}$ & $U L L$ & http://www.ull.es & \begin{tabular}{|l|} 
Universidad Politécnica \\
de Madrid \\
\end{tabular} & UPM & http://www2.upm.es \\
\hline Universidad de La Rioja & $U R$ & http://www.unirioja.es/ & Universitat Politècnica & UPV & http://www.upv.es \\
\hline $\begin{array}{l}\text { Universitat de les Illes } \\
\text { Balears }\end{array}$ & UIB & http://www.uib.es & & & \\
\hline & & & Fabra & UPF & http://www.upf.edu/ \\
\hline Palmas de Gran Canaria & ULPGC & http://www.ulpgc.es/ & Universidad Pública de & UPNA & http://www.unavarra.es/ \\
\hline Universidad de León & $U L$ & http://www.unileon.es/ & & & \\
\hline Universitat de Lleida & $U D L$ & http://www.udl.es/ & $\begin{array}{l}\text { Universidad Rey Juan } \\
\text { Carlos }\end{array}$ & URJC & http://www.urjc.es/ \\
\hline Universidad de Málaga & UMA & http://www.uma.es/ & Universitat Rovira $i$ & & \\
\hline Universidad de Murcia & UM & http://www.um.es/ & Virgili & URV & nстр://WWW.urv.es/ \\
\hline
\end{tabular}

Tabla 1. Universidades públicas españolas (fuente: Crue)

- ¿Hay alguna correlación entre la presencia web de las universidades españolas en Google Scholar y su producción académica?

- ¿Qué volumen de documentación presentan las universidades españolas a partir de sus dominios web en Google Scholar?

De esta forma, los objetivos principales son los siguientes:

- comparar la cobertura que proporciona Google Scholar de las universidades españolas con la mostrada por Scopus, y

- analizar la presencia y evolución de la documentación alojada en los dominios web de las universidades españolas.

Este trabajo se centra únicamente en las universidades públicas españolas, excluyendo a las privadas con el propósito de tratarlas de forma exclusiva en el futuro.

\section{Metodología}

\subsection{Correlación entre Scholar y Scopus}

La productividad académica de las universidades se extrae directamente de Scopus mediante los datos del ranking $\mathrm{Scimago}^{4}$, relativos a la producción total de las universidades públicas españolas (tabla 1), desde 2003 hasta 2007.

Para la publicación web, se consultan todos los dominios web de las universidades públicas españolas ${ }^{5}$ desde Scholar para el mismo período de tiempo. Este proceso se lleva a cabo en junio de 2008.

La consulta se realiza mediante el comando "site" al dominio principal de cada universidad. En el caso 
de la $U B$ y la $U D G$, se combinan los resultados de los dominios .es y .edu.

Finalmente, los datos de ambas series (Scopus y Scholar) se exportan a una hoja de cálculo, donde se obtiene la correlación entre ambas.

Se debe indicar que no se recuperan los artículos publicados por autores de una determinada universidad recogidos en Scholar, sino los artículos publicados en la parte pública del dominio web.

Esto significa que no se evalúa la producción científica de la universidad, sino más bien su compromiso de facilitar documentación científica a su comunidad mediante la Web y en concreto, de Scholar.

\subsection{Presencia y evolución}

El marco de trabajo se sitúa en el análisis de los dominios web de las universidades públicas españolas, desde enero hasta julio (ambos meses incluidos) de 2009.

Se recogen datos de todas las universidades públicas españolas, a excepción de la UPF, URV y UIA, de las que no se dispone de información debido a problemas técnicos ${ }^{6}$, por lo que no se consideran.

Los datos se obtienen de nuevo mediante la consulta directa a Scholar, pero sin ninguna restricción temporal. Posteriormente se exportan, durante la primera semana de julio, a una hoja de cálculo para ser tratados.

La toma de datos es mensual; se realizan varias catas por mes y se obtiene la media de dichas muestras. Esta operación se repite para cada dominio web de cada universidad, obteniendo un valor en bruto por mes. De éstos se obtiene la media mensual, denominada Scholar $(m)$, el recorrido y un valor llamado de representatividad relativa en Scholar (RScholar).

El recorrido se obtiene como la resta del valor último (junio) menos el primero (enero) e indica la ganancia o pérdida en los meses analizados.

$R S$ cholar se calcula normalizando de 0 a 100 los valores en bruto de cada dominio web para cada mes. Se otorga el valor 100 al dominio web de la universidad con valor máximo, y el resto se normalizan de forma proporcional. Finalmente, la media de los 6 valores normalizados (uno por mes) de cada dominio web proporciona el valor de RScholar.

Se recogen asimismo los datos relativos a la resta entre los valores totales en Google Scholar y los valores delimitados temporalmente a partir del año 2000 (en adelante Scholar recent, $G r$ ), para cada mes y dominio web.

De esta forma se premia la publicación web reciente de las universidades y no el simple volcado retrospecti-

\section{"Pese a que las posiciones no coinciden, 7 de las 10 primeras universidades en Scopus se encuentran igualmente entre las 10 primeras en Scholar"}

vo de material académico, que favorece a las universidades más antiguas al disponer de más documentación acumulada.

A partir de Google recent se obtiene la media de los 6 valores mensuales obtenidos, $G r(m)$, así como los correspondientes a su representatividad relativa $(R G r)$.

Finalmente, se indica que los resultados obtenidos no deben ser entendidos como aproximaciones a la calidad de las universidades o clasificaciones ordenadas donde un primer puesto signifique ser "mejor", sino simplemente como reflejo de lo que Google Scholar está mostrando de los dominios web académicos, síntoma de la buena o mala comunicación vía web que las universidades públicas españolas tienen con sus usuarios.

\section{Resultados}

\subsection{Scopus vs Scholar}

La tabla 2 muestra las diez primeras universidades públicas españolas en productividad en Scopus y Scholar para 2003-2007.

Pese a que las posiciones no coinciden, 7 de las 10 primeras universidades en Scopus se encuentran igualmente entre las 10 primeras en Scholar. Aun así, se observan ciertos comportamientos divergentes: mientras la $U A M, U S C$ y $U S$ pierden representatividad en Scholar, la $U D G$ y $U M$ la mejoran. La $U P C T, U H U$ y $U C O$ mejoran igualmente, aunque quedan fuera de las 10 primeras.

Los casos de la $U R$ y $U C M$ son especialmente reseñables pues la cantidad de publicación web recuperada en sus dominios web es mayor que la producción académica recogida por Scopus en el mismo período. Es más, la $U R$ aparece en el último puesto en productividad científica en Scopus mientras que se encuentra en primera posición en Scholar. Estos resultados se atribuyen, entre otros factores, a la indización de la base de datos Dialnet de la $U R$ por parte de Scholar.

El caso de la $U C M$ es diferente pues aun siendo la tercera universidad en producción científica, sus resultados en Scholar son desproporcionados debido a la indización de su servicio de sumarios Compludoc.

Por su parte, la figura 1 muestra la correlación entre los datos de Scholar y Scopus (dejando fuera de los cálculos a la $U R$ y $U C M$ por sus elevados valores en Scholar). 


\begin{tabular}{|c|r|c|r|}
\hline Universidades & Scopus & Universidades & Scholar \\
\hline$U B$ & 11.243 & UR & 30.700 \\
\hline$U A B$ & 9.364 & UCM & 16.300 \\
\hline$U C M$ & 8.911 & $U M$ & 1.550 \\
\hline$U A M$ & 7.851 & EHU & 1.490 \\
\hline$U V$ & 7.621 & $U B$ & 1.445 \\
\hline$U P C$ & 6.884 & $U G R$ & 1.280 \\
\hline$U G R$ & 5.774 & $U A B$ & 1.070 \\
\hline$U S C$ & 5.097 & $U P C$ & 778 \\
\hline$U S$ & 4.927 & $U D G$ & 671 \\
\hline$E H U$ & 4.675 & UV & 661 \\
\hline
\end{tabular}

Tabla 2. Productividad (Scopus) y publicación web (Scholar) de las 10 primeras universidades públicas españolas (2003-2007)

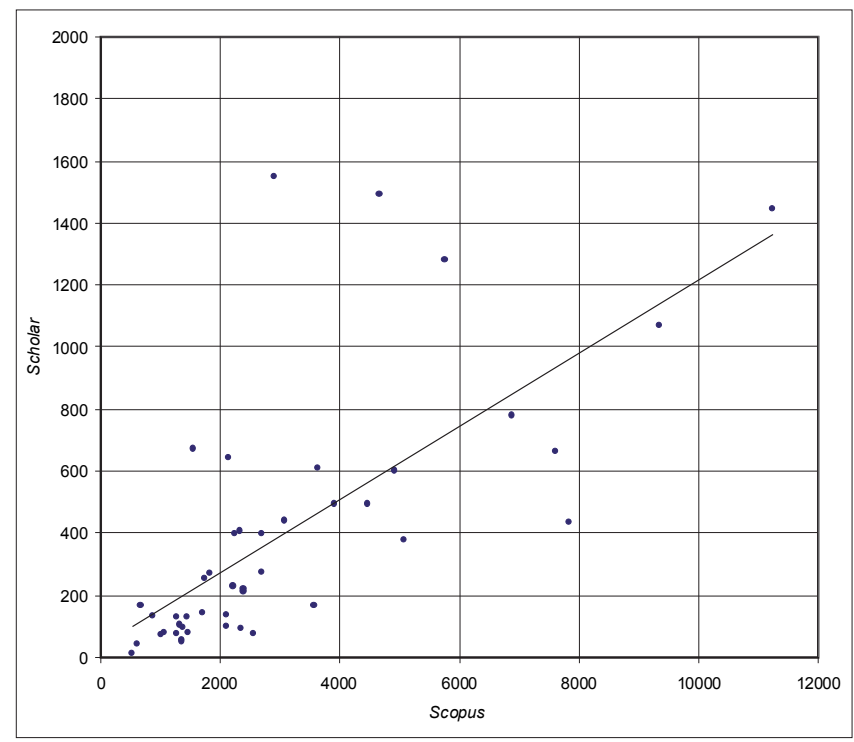

Figura 1. Correlación entre Scholar y Scopus (2003-2007)

Se obtiene una curva de regresión positiva, es decir, la mayoría de las universidades más productivas en Scopus lo son igualmente en Scholar, con un coeficiente de correlación de Pearson de 0,70 (si se tienen en cuenta $U R$ y $U C M$ resulta un coeficiente de sólo 0,08 ).

\subsection{Producción y publicación web}

La tabla 3 muestra los 46 dominios web estudiados, ordenados de forma descendente según su valor de RScholar. Así mismo se muestran los valores de representatividad extrayendo del cálculo a la $U R$ y $U C M$ (RScholar*) y la media de los valores brutos mensuales. Completan la tabla los valores de recorrido, $R G r$ y $\operatorname{Gr}(m)$.

La $U R$ y $U C M$ aparecen las primeras en el ranking de RScholar, a una distancia considerable del resto. El dominio de la primera, de hecho, es abrumador incluso respecto a la $U C M$. Tras estas dos universidades, el resto aparece con valores más similares.
Respecto a $R G r$, los resultados muestran que 31 universidades (de 46) mejoran su representatividad al tener en cuenta únicamente la publicación web reciente, entre ellas las 17 primeras del ranking RScholar. Destaca la $U C M$ (que incrementa cerca del 35\% de representatividad total), así como la $U M, E H U$ y $U D G$.

Si observamos el recorrido, vemos caídas de la $U B$ (5.540 documentos menos que en enero), $U M$ y $U B$. En cuanto a dominios web de universidades con recorrido positivo, destacan UGR y UJI. Así mismo, merecen atención los comportamientos de la $U V A$ y $U L$ (sin apenas cambios), y de la $U D C$ (crecimiento 0 ).

Es igualmente de interés la relativamente buena posición de las universidades politécnicas, con dos entre las 10 primeras ( $U P C$ y $U P M)$, y la $U P V$ cerca, en la decimosegunda posición.

En la figura 2 se muestra la distribución-exponencial, o de larga cola-, de la media de registros por universidad.

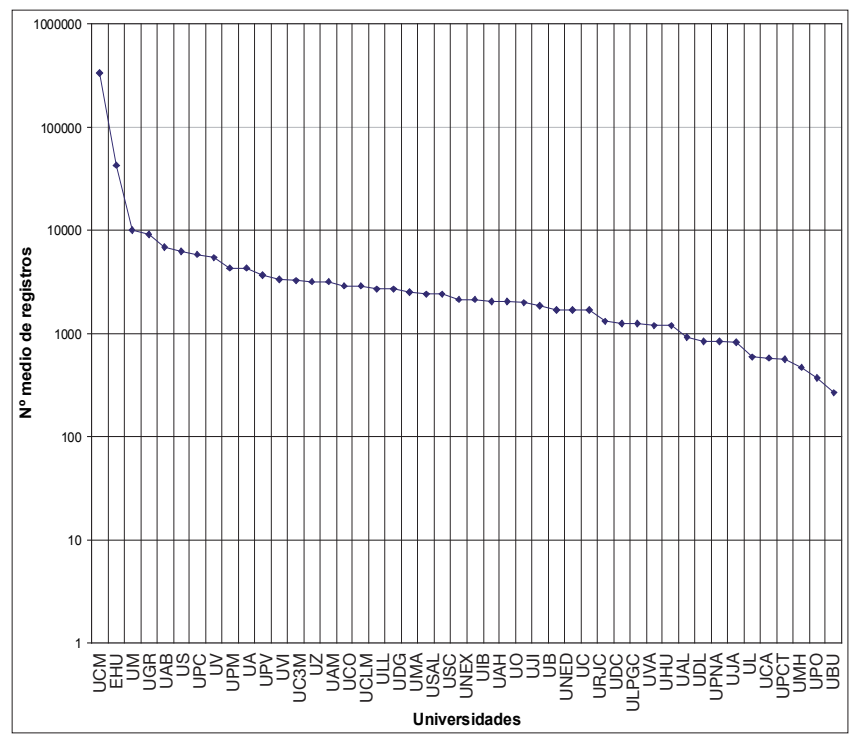

Figura 2. Distribución del número medio de registros por universidad (enero-junio 2009)

\subsection{Evolución}

En la figura 3 se detalla la evolución del tamaño de las 10 primeras universidades, a excepción de la $U R$ y UCM.

En términos generales, $U M, E H U$ y $U G R$ sobresalen respecto al resto. Todos los dominios web, no obstante, presentan unas tendencias estables y ligeramente positivas durante todos los meses estudiados a excepción de marzo, donde se producen cambios importantes en prácticamente todos los dominios web, que determinan en buena medida los valores de recorrido obtenidos. Destaca el crecimiento de la $U G R, U S, U V$ y UA, y las caídas en la $U M, U A B$ y $U P C$. 


\begin{tabular}{|c|c|c|c|c|c|c|}
\hline Universidades & RScholar & RScholar* & Scholar(m) & Recorrido & RGr & $\operatorname{Gr}(\mathbf{m})$ \\
\hline$U R$ & 100,00 & - & 334.000 & 55.000 & 100,00 & $48.600,00$ \\
\hline UCM & 12,82 & - & 42.450 & -2.300 & 47,78 & $19.450,00$ \\
\hline EHU & 3,03 & 98,58 & 10.087 & 380 & 6,00 & $2.561,70$ \\
\hline$U M$ & 2,74 & 88,35 & 9.048 & -1.900 & 9,37 & $4.000,00$ \\
\hline$U G R$ & 2,07 & 67,68 & 6.922 & 1.140 & 4,02 & $1.795,00$ \\
\hline$U A B$ & 1,88 & 60,75 & 6.218 & -730 & 3,31 & $1.426,70$ \\
\hline US & 1,75 & 56,97 & 5.828 & 600 & 1,87 & 828,50 \\
\hline$U P C$ & 1,65 & 53,59 & 5.485 & -370 & 2,47 & $1.050,00$ \\
\hline$U V$ & 1,31 & 42,63 & 4.360 & 740 & 1,99 & 893,50 \\
\hline UPM & 1,29 & 42,14 & 4.312 & 430 & 1,57 & 764,50 \\
\hline$U A$ & 1,11 & 36,17 & 3.698 & 830 & 1,15 & 528,83 \\
\hline$U P V$ & 0,99 & 32,26 & 3.300 & 830 & 1,64 & 720,83 \\
\hline UVI & 0,97 & 31,77 & 3.250 & 410 & 1,27 & 536,00 \\
\hline UC3M & 0,95 & 31,04 & 3.175 & 50 & 1,44 & 641,33 \\
\hline$U Z$ & 0,95 & 30,86 & 3.157 & 390 & 1,40 & 623,17 \\
\hline UAM & 0,88 & 28,57 & 2.922 & 450 & 1,26 & 573,17 \\
\hline UCO & 0,87 & 28,48 & 2.913 & 480 & 2,22 & 947,50 \\
\hline UCLM & 0,81 & 26,44 & 2.705 & 210 & 0,66 & 299,33 \\
\hline$U L L$ & 0,80 & 26,22 & 2.682 & 480 & 0,78 & 336,83 \\
\hline$U D G$ & 0,75 & 24,51 & 2.505 & 480 & 2,66 & $1.208,20$ \\
\hline UMA & 0,73 & 23,60 & 2.415 & -80 & 1,25 & 550,83 \\
\hline USAL & 0,71 & 23,30 & 2.383 & 280 & 0,87 & 399,00 \\
\hline USC & 0,64 & 21,00 & 2.148 & 250 & 1,32 & 580,00 \\
\hline UNEX & 0,64 & 20,94 & 2.142 & 410 & 0,46 & 197,67 \\
\hline UIB & 0,61 & 19,86 & 2.032 & 250 & 0,85 & 382,00 \\
\hline$U A H$ & 0,61 & 19,74 & 2.020 & 130 & 0,61 & 279,17 \\
\hline UO & 0,60 & 19,66 & 2.012 & 200 & 0,60 & 267,50 \\
\hline UJI & 0,55 & 18,22 & 1.863 & 1.200 & 0,98 & 519,17 \\
\hline$U B$ & 0,53 & 16,34 & 1.678 & -5.540 & 0,98 & 316,50 \\
\hline UNED & 0,51 & 16,69 & 1.707 & 180 & 1,01 & 467,67 \\
\hline$U C$ & 0,51 & 16,62 & 1.700 & 100 & 0,27 & 122,33 \\
\hline URJC & 0,39 & 12,68 & 1.297 & 320 & 0,34 & 154,50 \\
\hline$U D C$ & 0,37 & 12,07 & 1.235 & 0 & 0,40 & 174,50 \\
\hline ULPGC & 0,37 & 12,16 & 1.243 & 361 & 0,24 & 106,50 \\
\hline UVA & 0,36 & 11,64 & 1.192 & 10 & 0,22 & 99,83 \\
\hline$U H U$ & 0,36 & 11,67 & 1.193 & 270 & 0,31 & 150,67 \\
\hline$U A L$ & 0,28 & 8,97 & 919 & -190 & 0,23 & 111,00 \\
\hline$U D L$ & 0,26 & 8,25 & 845 & -132 & 0,30 & 121,00 \\
\hline UPNA & 0,25 & 8,17 & 836 & 65 & 0,16 & 67,50 \\
\hline UJA & 0,25 & 7,99 & 817 & 42 & 0,33 & 146,67 \\
\hline$U L$ & 0,18 & 5,83 & 597 & -13 & 0,21 & 95,33 \\
\hline$U C A$ & 0,17 & 5,64 & 577 & 98 & 0,10 & 50,00 \\
\hline UPCT & 0,17 & 5,55 & 567 & 479 & 0,32 & 170,33 \\
\hline$U M H$ & 0,14 & 4,53 & 463 & 19 & 0,34 & 138,83 \\
\hline UPO & 0,11 & 3,60 & 368 & 88 & 0,09 & 41,33 \\
\hline$U B U$ & 0,08 & 2,61 & 268 & 27 & 0,05 & 20,83 \\
\hline
\end{tabular}

Tabla 3. Valores medios brutos y de representatividad de la producción (Scopus) y publicación web (Scholar) por universidad (enero-junio 2009). Algunas variables se han redondeado para mayor claridad.

Según la última edición del ranking web (enero2008), la $U M$ y $E H U$ son las universidades con mayor tamaño en Scholar ( $\sin$ contar a la UR y UCM), logrando las posiciones 71 y 94 a escala mundial respectivamente. Estos datos concuerdan con los resultados obtenidos, teniendo en cuenta la caída de la $U M$ en marzo.

La figura 4 muestra por su parte la evolución de las 10 primeras universidades (excluyendo a la $U R$ y $U C M$ ) en Scholar recent, destacando de nuevo el mes de marzo como momento de cambios, con crecimientos muy notables para la $U M, U G R, U D G$ y $U P M$.

De nuevo $U M$ (en primera posición), $E H U$ y $U G R$ son las universidades con más registros. Aun así, sorprende la aparición en los primeros puestos de la $U D G$ y $U C O$ así como la pérdida de puestos de la $U A$ y $U V I$, que abandonan el top ten en publicación web, al que llega la $U P V$ gracias a su importante incremento en mayo. 


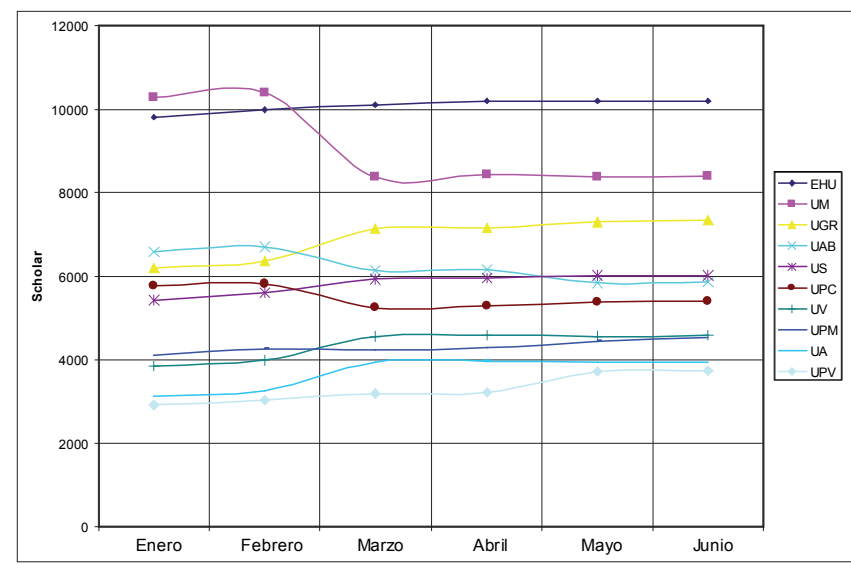

Figura 3. Evolución de los dominios web académicos españoles en Scholar (enero-junio 2009)

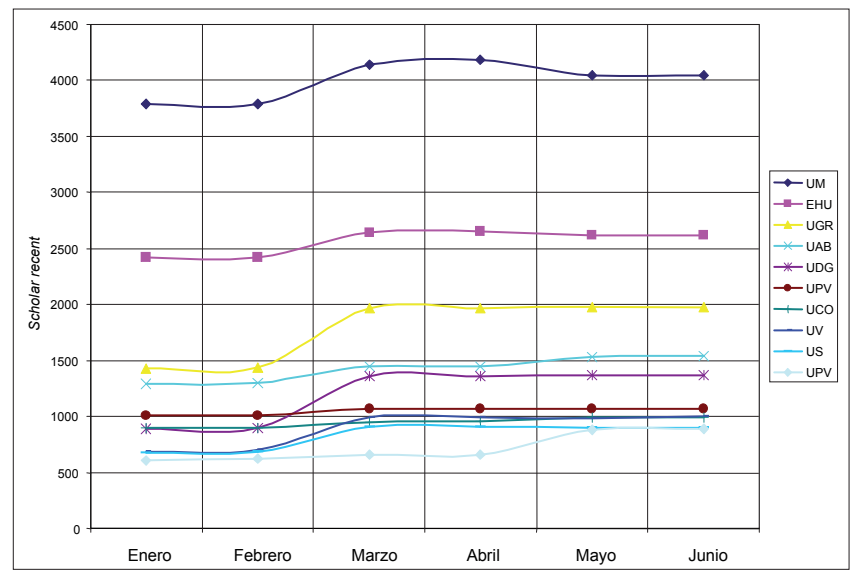

Figura 4. Evolución de los dominios web académicos españoles en Scholar recent (enero-junio 2009)

\section{Discusión}

Pese a obtener una correlación positiva (coeficiente de Pearson de 0,70) entre la productividad en Scopus y la publicación web en Scholar de las distintas instituciones (exceptuando a la $U R$ y $U C M$ ), se asume la necesidad de una corrección futura por tamaño de universidad, aunque este parámetro no puede ser calculado de forma precisa pues no es proporcionado por todas las universidades, resultando además un término difuso y sin definición clara.

Existen grandes diferencias en los resultados brutos totales, que se asocian a:

- La recuperación tanto de artículos académicos no producidos por la universidad, como documentos no académicos, resúmenes, fichas catalográficas, etc. Muestra de ello es la indización de Dialnet o Compludoc.

- Diferentes políticas en cada dominio web de universidad en relación con el Open Access, que provoca que ciertas universidades queden mal representadas en Scholar aun disponiendo de suficiente material académico producido y publicado, como por ejemplo la $\mathrm{USC}^{7}$, que pese a ser de las universidades con más producción, todavía no dispone de un repositorio institucional.

\section{"La UR y UCM aparecen las primeras en el ranking de $R S c h o l a r$, a una distancia considerable del resto"}

- Problemas de Scholar en la indización de algunos repositorios institucionales, donde se recopilan metadatos de los proveedores de servicios y no de los repositorios directamente, restando por tanto visibilidad a los dominios web de universidades.

Este es el caso -entre otros- de la UAM, que si bien dispone de un repositorio institucional ${ }^{8}$, su contenido no se encuentra disponible dentro del dominio web y, por tanto, no es recogido por la metodología seguida en este trabajo, lo que explica las diferencias entre producción científica y publicación web obtenidas para esta institución.

No es el propósito de este trabajo identificar todas las universidades que se encuentran en esta situación, aunque se asume que la posición de éstas mejoraría si solucionaran este problema, pues se considera una mala práctica ${ }^{9}$ la disposición de documentación académica fuera del dominio web institucional.

\section{Conclusiones}

El trabajo muestra una comparación entre la publicación web, recuperada por Scholar mediante los documentos alojados dentro de los dominios web de cada universidad pública española, y su productividad académica medida desde Scopus.

Los resultados demuestran objetivamente que, sin tener en cuenta los dominios web de universidades con grandes servicios de referencia (Dialnet y Compludoc), la producción académica en Scopus y la publicación web en Scholar están correlacionadas, aunque son conjuntos documentales de diferente naturaleza.

Pese a la curva positiva de regresión obtenida, la posible interrelación entre productividad en Scopus y publicación web en Scholar precisa de un análisis más profundo en el futuro, debido a la dependencia de variables externas no controlables como el filtrado de documentos y los métodos de indización de los repositorios institucionales (por parte de Scholar) o el tamaño y las políticas de acceso abierto (por parte de las universidades).

Pese a todo, las ventajas de Scholar como herramienta de búsqueda de información y como escaparate de atracción de usuarios para las universidades son innegables. 
Por ello, tal vez las acciones emprendidas por la $U R$ y $U C M$ disfracen la verdadera actividad en publicación web de dichas universidades en Scholar (en todo caso, son carencias en el filtrado de esta herramienta), pero generan una presencia y visibilidad internacional que no logra ninguna otra universidad española.

Por otra parte, sólo 9 de los 46 dominios web universitarios analizados presentan en junio valores más bajos que en enero (recorrido negativo), lo que muestra un crecimiento generalizado, aunque moderado.

Los recorridos negativos (siendo destacable el caso de la $U B$ ) pueden deberse a la eliminación de cierta documentación temporal o cambios en las políticas web (como mayor uso de la intranet o de diferentes dominios web, consideradas ambas malas prácticas ${ }^{9}$ ).

Respecto a Scholar recent, los primeros puestos del ranking nacional no presentan muchas variaciones respecto a Scholar, aunque sí se observan diferencias en los valores de representatividad relativa. $U C M$ es la que registra un mayor crecimiento y, en menor medida, $E H U, U M, U G R, U D G$ y $U C O$.

En cuanto a trabajos futuros de investigación, se estima conveniente la comparación de los resultados obtenidos con los correspondientes a las universidades españolas privadas así como con algunas extranjeras, con el fin de contextualizarlos de forma más precisa.

Asimismo, también resultaría de interés el futuro análisis cuantitativo de los diferentes tipos documentales cubiertos en Scholar por dominio web de universidad.

\section{Agradecimientos}

Los autores agradecen enormemente el excelente trabajo realizado por los revisores, que con sus consideraciones y sugerencias han ayudado a mejorar notablemente el texto.

\section{Notas}

1. Ranking web de universidades del mundo http://www.webometrics.info/

2. Web popularity ranking

http://www.4icu.org/top200/

3. Ranking universitario de transparencia web

http://www.universidad.edu.co

4. Ranking de universidades españolas por calidad investigadora, publicado por Scimago a partir de los datos extraídos de Scopus.

http://www.magisnet.com/pdf/RankingEspanyolUniversidades.pdf

5. Conferencia de Rectores de las Universidades Españolas (CRUE) http://www.crue.org/

6. No se dispone de los datos relativos a enero debido a errores en las queries de consulta y en ningún caso hay problemas en dichos dominios para la extracción de información.

7. http://www.accesoabierto.net/repositorios

8. http://digitool-uam.greendata.es/R

9. http://www.webometrics.info/best_practices.html

\section{Referencias}

Aguillo, Isidro F. "Hacia un concepto documental de sede web". El profesional de la información, 1998, v. 7, n. 1-2, pp. 45-46.

Aguillo, Isidro F.; Ortega, José-Luis; Fernández, Mario. "Webometric ranking of world universities: introduction, methodology and future developments". Higher education in Europe, 2008, v. 33, n. 2/3, pp. 234-244.

Aguillo, Isidro F.; Granadino, Begoña; Ortega, José-Luis; Prieto, JoséAntonio. "Scientific research activity and communication measured with cybermetric indicators". Journal of the American Society for Information Science and Technology, 2006, v. 57, n. 10, pp. 1296-1302.

Bar-Ilan, Judit. "A microscopic link analysis of academic institutions within a country - the case of Israel". Scientometrics, 2004, v. 59, n. 3, pp. 391-403.

Harzing, Anne-Wil K.; Van der Wal, Ron. "Google Scholar as a new source for citation analysis". Ethics in science and environmental politics, 2008, v. 8, n. 1, pp. 61-73.

Jacsó, Péter. "Google Scholar revisited". Online information review, 2008a, v. 32, n. 1, pp. 102-114.

http://www.jacso.info/PDFs/jacso-GS-revisited-OIR-2008-32-1.pdf

Jacsó, Péter. "The pros and cons of computing the h-index using Google Scholar". Online information review, 2008b, v. 32, n. 3, pp. 437-452.

http://www.jacso.info/PDFs/jacso-pros-and-cons-of-computing-the- $h$ index.pdf

Kousha, Kayvan; Thelwall, Mike. "Sources of Google Scholar citations outside the science citation index: a comparison between four science disciplines". Scientometrics, 2008, v. 74, n. 2, pp. 273-294.

Mayr, Philipp; Walter, Anne-Kathrin. "An exploratory study of Google Scholar". Online information review, 2007, v. 31, n. 6, pp. 814-830.

http://www.ib.hu-berlin.de/ mayr/arbeiten/OIR31-6.pdf

Neuhaus, Chris; Neuhaus, Ellen; Asher, Alan; Wrede, Clint. "The depht and breadth of Google Scholar: an empirical study". Libraries and the academy, 2006, v. 6, n. 2, pp. 127-141.

Noruzi, Alireza. "Google Scholar: the new generation of citation indexes". Libri, 2005, v. 55, n. 4, pp. 170-180.

http://www.librijournal.org/pdf/2005-4pp170-180.pdf

Payne, Nigel; Thelwall, Mike. "A statistical analysis of UK academic web links". Cybermetrics, 2004, v. 8, n. 1 .

http://www.cindoc.csic.es/cybermetrics/articles/v8ilp2.html

Smith, A.; Thelwall, Mike. "Web impact factor for Australasian universities". Scientometrics, 2002, v. 54, n. 3, pp. 363-380.

Torres-Salinas, Daniel; Ruiz-Pérez, Rafael; Delgado-López-Cózar, Emilio. "Google Scholar: ¿una herramienta para la evaluación de la ciencia?". Anuario ThinkEPI 2010 [en prensa].

http://www.thinkepi.net/google-scholar-\%C2\%BFuna-herramienta-parala-evaluacion-de-la-ciencia

Enrique Orduña-Malea, Facultad de Informática, Universidad Politécnica de Valencia (UPV). Camino de Vera $s / n, 46022$ Valencia

enorma@fiv.upv.es

Jorge Serrano-Cobos, MASmedios, Álvaro de Bazán 10, 46010 Valencia

jorgemasmedios.com

jorgeserrano@gmail.com

Núria Lloret-Romero, Universidad Politécnica de Valencia $(U P V)$, Área de Biblioteca y Documentación Científica, Camino de Vera, s/n, 46022 Valencia nlloret@upvnet.upv.es 